\title{
Detecting phonemes and letters in text: Interactions between different types and levels of processes
}

\author{
VIVIAN I. SCHNEIDER and ALICE F. HEALY \\ University of Colorado, Boulder, Colorado
}

\begin{abstract}
In six experiments, subjects detected phonemes or letters in text presented auditorily or visually. Experiments 1 and 2 provided support for the hypothesis that a mismatch between the phoneme and letter representations of a target leads to detection errors. In addition, visual word unitization processes were implicated. Experiments 3 and 4 provided support for the hypothesis that the Gestalt goodness of pattern affected detection errors when subjects searched for letters. Experiments 5 and 6 demonstrated that the effects of unitization on the detection of letters in common words were decreased by altering the familiar configuration of the test words. The combined results of all six experiments lead to the conclusion that both visual and phonetic processes influence letter detection, that these processes communicate through a type of cross-checking, and that there are at least two levels of visual (and perhaps of phonetic) processing involved in the letter detection task.
\end{abstract}

What is the relationship between the phonetic and graphemic representations of words in the mental lexicon? In previous work, Schneider, Healy, and Gesi (1991) suggested that both phonemes and letters are represented for a given word in the mental lexicon and that there is a crosschecking or communication between these two types of representations. Two different levels of processing-letter and word-and two different types of processing-visual and phonetic-were found to contribute to the crosschecking procedure.

The letter-phoneme cross-checking procedure proposed by Schneider et al. (1991) is consistent with some popular models of the reading process (see, e.g., LaBerge \& Samuels, 1974) and with experimental studies in which both visually and auditorily presented text have been used. For example, it has been shown that phonetic processes affect the categorization of visually presented words (see, e.g., Van Orden, 1987), and there is an enhanced wordsuperiority effect for silent letters in words (see Krueger, 1992). Further, it has been shown that orthographic processes affect the monitoring of auditorily presented words (see, e.g., Seidenberg \& Tanenhaus, 1979), and speech perception is affected by the visual presentation of the speaker's lip movements (see, e.g., McGurk \& Mac-

\footnotetext{
This research was supported in part by Army Research Institute Contract MDA903-90-K-0066 to the Institute of Cognitive Science at the University of Colorado. Thanks are extended to David Mason, David McCain, Danielle McNamara, and Ursula Williams for their help in testing subjects in these experiments. We also thank Seth Greenberg, Robert Proctor, and two anonymous reviewers for their helpful comments concerning earlier versions of the manuscript. Correspondence should be addressed to V. I. Schneider, Department of Psychology, University of Colorado, Campus Box 345, Boulder, CO 80309-0345.
}

Donald, 1976; see also Massaro, 1987, for an expanded discussion of the integration of auditory and visual information in speech perception). Schneider et al. (1991) used a detection task in which subjects read or listened to text and wrote down every word containing a given target, which was either a phoneme or a letter. It was found that the subjects were particularly likely to make detection errors when there was a mismatch between the phoneme and letter representations of the target in a given word. In the present experiments, we used the same paradigm to provide converging evidence for the cross-checking between phoneme and letter representations of words in the mental lexicon.

Unitization processes have also been found to influence detection errors when text is presented visually, but not when it is presented auditorily (Schneider, Healy, Ericsson, \& Bourne, 1989). Specifically, it has been found that subjects reading printed text are particularly likely to make letter detection errors when the letters occur in common words that form familiar visual configurations (see, e.g., Healy, 1976). According to a unitization account of these findings (see, e.g., Healy, Oliver, \& McNamara, 1987), the processing of text occurs in parallel at different levels, and familiarity of a unit at a given level facilitates its processing. The additional assumption is made that when a unit is identified at a given level, processing of all the component units is terminated, and the reader moves on to the next segment of text. For example, according to the unitization account, processing occurs in parallel at the level of the letter and the level of the word. After a word has been identified, processing of all of the component letters in the word is terminated, and the subject moves on to the next word. By this account, letter detection errors often occur with common words because the 
subject has identified the word before identifying the target letter in it.

The present experiments also allow for a comparison of the relative importance to letter detection of the letterphoneme cross-checking processes and the visual unitization processes. Unitization processes are not the only visual processes affecting letter detection. Other relevant visual processes concern the location of the target letter in the word (see, e.g., Corcoran, 1966, and Healy, 1976, for earlier discussions of this issue) and the Gestalt "goodness" of the target patterns (see, e.g., Garner, 1974; Garner \& Clement, 1963). These other visual processes, which operate at the level of the individual letter within the word, are compared in the present experiments with the unitization processes, which operate at the level of the word. More generally, the experiments permit us to develop a picture of the interactions between different types of processes (visual and phonetic) as well as the interactions between different levels of processes (letter and word) in the letter detection task.

As mentioned above, the specific interactions between different types and different levels of processes envisioned by Schneider et al. (1991) involved a cross-checking procedure that is sensitive to a mismatch between the letter and phoneme representations of a target in a word. That is, the cross-checking procedure is sensitive to whether the visual shape of the target is consistent with the phoneme code for the target. For example, subjects miss the target in the word of when searching for the letter $f$ because there is a mismatch between the letter $f$ and the phoneme $/ \mathrm{v} /$ in the lexical entry for the word of. This cross-checking procedure may be understood in activation terms. Activity from the letter $f$ and the phoneme /f/ may excite one response unit, and activity from the letter $v$ and the phoneme $/ \mathrm{v} /$ may excite another response unit. For the word of (which contains the letter $f$ pronounced as the phoneme $/ \mathrm{v} /$ ), activity from the letter $f$ may inhibit activity for the response unit associated with the letter $v$, and activity from the phoneme $/ \mathrm{v} /$ may inhibit activity associated with the phoneme /f/. Hence, there may not be sufficient excitation from the combined sources to initiate either response unit, so that subjects will fail to detect the target.

The aim of Experiments 1 and 2 was to provide converging evidence for this cross-checking procedure and for visual word unitization processes. Subjects searched for either the phoneme $/ v /$ or the phoneme / $/$ / in text presented visually (Experiment 1) or auditorily (Experiment 2). If subjects are indeed using the cross-checking procedure, they should make more errors on of than on other words when searching for the phoneme / $/ \mathrm{w}$ with text presented either auditorily or visually. If visual but not auditory word unitization processes affect phoneme detection, subjects should also make more errors on of than on other words when searching for the phoneme / $/$ / with text presented visually but not with text presented auditorily.

The aim of Experiments 3 and 4 was to compare the impact on letter detection of visual word unitization pro- cesses and other visual processes, including the location of the target letter and the Gestalt goodness of the target patterns. Subjects searched visually presented text for either the letter $o$ or the letter $t$ with either the test word or the target location in the test word controlled. To assess the effects of visual word unitization processes, the test words to and $t w o$, which have the same pronunciation but differ greatly in familiarity, were compared. To assess the effects of target location, the test words to and not, which have different orders of the target letters, were compared. In Experiment 3, we employed a standard font in which the letter $o$ has well-formed Gestalt properties of its configuration and the letter $t$ is less regular and symmetrical. If word unitization affects letter detection, subjects should make more errors on to than on two. If the Gestalt goodness of pattern affects letter detection, subjects should make fewer errors on the letter $o$ than on the letter $t$. On the other hand, if the location of the target letter is crucial, the pattern of detection errors should depend on the relative locations of the target letters. In Experiment 4 , we employed an unusual font that disturbs the Gestalt goodness of pattern for the letter $o$ and improves it for the letter $t$. If the Gestalt goodness of pattern affects letter detection, detection error rates on the letter $o$ should increase and those on the letter $t$ should decrease.

Experiments 5 and 6 also involved manipulations of type font, in this case to vary the familiar configuration of common test words (of and the) as well as the configuration of the individual target letters. In each experiment, a standard font was compared with a script font that altered the familiar configuration of the test words and disturbed the Gestalt goodness of pattern for the letter $o$. Subjects in Experiment 5 searched for either the letter $o$ or the letter $f$. Subjects in Experiment 6 searched for the letter $t$. If word unitization affects letter detection, the detection error rate on the common words of and the should be lower with the script font relative to that with the standard font. In addition, if the Gestalt goodness of pattern affects letter detection, the decrease in detection errors for the letter $o$ on the word of due to disturbing the word configuration should be offset to some extent by an opposing increase in detection errors due to disturbing the letter configuration. Finally, if subjects are indeed using the crosschecking procedure, the detection error rate on the word of should also be increased to some extent for the target $f$ relative to that for the target $o$ with both fonts.

\section{EXPERIMENT 1}

As summarized above, Schneider et al. (1991) suggested that in reading a passage and searching for a target letter or phoneme, subjects make detection errors when there is a mismatch or discrepancy between the phoneme and letter representations of the target in a word. This suggestion was prompted by the observation that subjects make many detection errors on the word of when searching for the letter $f$, because there is a mismatch between 
the letter $f$ and the phoneme $/ \mathrm{v} /$ in the lexical representation. It follows from this suggestion that subjects should also make many detection errors on the word of when searching for the phoneme $/ \mathrm{v} /$ instead of the letter $f$, because the mismatch between the phoneme and letter representations of the target would still be present. In fact, Schneider et al. found that subjects made a disproportionately large number of detection errors on of when searching through a passage presented either visually or auditorily for the combined target phonemes $/ \mathrm{f} /$ and $/ \mathrm{v} /$. However, it remained to be seen whether subjects would show the same pattern of results when given only the single target $/ \mathrm{v} /$. It is possible that the pattern would be evident only with the combined target, not with the single target $/ \mathrm{v} /$, if the mismatching effect should be found to rely on a comparison of the two targets. This pattern of results would also be of particular interest for the single target $/ \mathrm{v} /$, because it would be inconsistent with the original explanation that had been proposed by Read (1983) for the finding that subjects searching for the letter $f$ in visually presented text made many errors when the $f$ occurred in the word of. According to Read, subjects missed the $f$ in of because they were scanning a phonologically recoded version of the text and searching exclusively for the phoneme /f/, and of is the only word among the 20,000 most common in which the letter $f$ does not correspond to the phoneme /f/ (Venezky, 1970). According to this explanation, there is no reason for subjects searching for the phoneme / $/$ to make an error on the word of, because in this case the phoneme for which subjects are searching $(/ \mathrm{v} /)$ does occur in the test word. The purpose of Experiment 1 was to examine this question.

Our subjects read passages and searched for target phonemes. All materials (including instructions regarding the target phonemes) were presented visually. The subjects wrote down any words containing the target phoneme in a space below the passage, as in the earlier studies by Schneider et al. (1989; Schneider et al., 1991). In the critical condition, the subjects were given the consonant phoneme $/ \mathrm{v} /$ as the target. In a control condition, the subjects were given the schwa vowel phoneme /a/ as the target. There is no mismatch between the letter and phoneme representations of the target in the word of for the vowel $/ 2 /$. Also, Schneider et al. (1991) found a much smaller tendency to make detection errors on of with the letter $o$ than with the letter $f$ as the target, and $\operatorname{Read}(1983)$ found essentially no detection errors on of when subjects searched for the letter $o$ rather than the letter $f$. However, in the study by Schneider et al. (1991), the proportion of errors on of was significantly greater than the proportion of errors on other words even with the $o$ target. The small but significant effect with the letter $o$ was attributed by Schneider et al. (1991) to visual word unitization processes. Hence, it was predicted that subjects in the present experiment would make a significantly greater proportion of errors on of than on other words when searching for either the vowel phoneme $/ 2 /$ or the consonant phoneme $/ \mathrm{v} /$, and that this effect of test word would be greater for the consonant than for the vowel phoneme.

\section{Method}

Subjects. Fifty-six undergraduate students taking a course on cognitive psychology participated as subjects on the Ist day of their course before the presentation of any material on cognitive psychology. There were equal numbers of subjects in the two counterbalancing groups. All were native speakers of English who had not participated in similar experiments previously. Sixteen additional subjects were tested, but their data were not analyzed; 2 of these additional subjects had seen the instructions from another condition, 12 of them made two or more false positive responses, and the remaining 2 were randomly selected to be dropped, in order to equate the numbers of subjects in each group. False positive responses were usually exceedingly rare in our previous letter detection experiments (see, e.g., Schneider et al., 1989). The large number of false positives by some subjects in the present experiment suggested that those subjects were confused about the definition of the schwa phoneme.

Materials. Two 26-word passages were constructed, each of which included two instances of the word of and four other words that contained both of the target phonemes (Passage A: shoveling, gloves, lovely, above; Passage $\mathrm{B}$ : doves, shoving, above, lovers). There were no other instances of either target phoneme. Passage A was, "That lonely man while shoveling snow found white gloves and old photos of two lovely giris both of whom rented small rooms right above his own." Passage B was, "That large flock of doves, after lots of pushing and shoving, finished eating and then flew high above those parked cars, scaring lovers who were inside."

The passages and instructions were typed with Courier 12-pitch font produced by a laser printer connected to a VAX computer.

Procedure. The subjects were tested in two large groups. Each subject participated in two conditions; the order of conditions was counterbalanced across subjects. The conditions differed only in the target sound. In the consonant condition, the target was the sound $/ \mathrm{v} /$ (as the final sound in the word love). In the vowel condition, the target was the sound /a/ (as the vowel or middle sound in the word love). The subjects in both counterbalancing groups were shown Passage A first, followed by Passage B, so that across subjects each passage was used equally often in each condition.

An instruction sheet preceded each passage. The subjects were instructed to read the passage as fast as they could, but any time they came to a word that contained the target sound, they were to write that word in the space below the dotted line, which was beneath the passage. They were also told that if they realized that they had missed a word with the target sound in it, they should not go back to that word. Further, they were reminded that the sound could occur anywhere in the word.

Design. The design was a $2 \times 2 \times 2$ mixed factorial. The first factor, target order (vowel first, consonant first), was a counterbalancing factor that was varied between subjects. The other two factors, target (consonant, vowel) and test word (of, other), were varied within subjects.

\section{Results}

The results are summarized in Table 1 in terms of the proportion of times out of the number possible that the subjects missed a target phoneme as a function of target and test word. Table 1 does not include a breakdown by the between-subjects counterbalancing factor of target order, because it did not enter into any significant main effect or interactions. As in previous experiments, there was a large increase in detection errors for of relative to 
Table 1

Proportion of Targets Missed in Experiment as a Function of Target and Test Word Test Word

\begin{tabular}{ccc} 
Target & of & Other Words \\
\hline $1 \mathrm{v} /$ & .777 & .027 \\
$12 /$ & .500 & .040 \\
\hline
\end{tabular}

other words. Also, more errors were made with the consonant than with the vowel target. Most importantly, the difference between of and other words was diminished, but still sizable, with the vowel target. A mixed analysis of variance yielded significant main effects of test word $\left[F(1,54)=175.1, M S_{\mathrm{e}}=0.1171, p<.001\right]$ and target $\left[F(1,54)=21.1, M S_{\mathrm{e}}=0.0460, p<.001\right]$, as well as a significant interaction of those two factors $[F(1,54)=$ 25.2, $\left.M S_{\mathrm{e}}=0.0467, p<.001\right]$.

Separate planned analyses were conducted on the data for each target. The effect of test word was significant both for the consonant target $\left[F(1,54)=248.7, M S_{\mathrm{e}}=\right.$ $0.0633, p<.001]$ and for the vowel target $[F(1,54)=$ $\left.59.0, M S_{\mathrm{e}}=0.1004, p<.001\right]$.

\section{Discussion}

On the basis of the suggestion by Schneider et al. '(1991) that the mismatch between the phoneme and letter representations of a target leads to detection errors, it was predicted that subjects would make many errors on the word of when searching for the phoneme $/ \mathrm{v} /$, just as it had been shown previously that they make many errors on of when searching for the letter $f$. Indeed, we found that the subjects, when searching for the consonant phoneme $/ \mathrm{v} /$, missed more than $75 \%$ of the ofs but less than $3 \%$ of the other test words. This finding (which was obtained with the single target $/ v /$ ) is not consistent with the possibility (based on the similar finding obtained previously when subjects searched for the combined targets $/ \mathrm{f} /$ and $/ \mathrm{v} /$ ) that the mismatching effect relies on a comparison of two targets. This finding is also inconsistent with the original explanation proposed by Read (1983) for the finding that subjects searching for the letter $f$ make an exceptionally large number of errors on the word of. According to that explanation, subjects scan a phonologically recoded version of the text for the phoneme /f/ and miss the $f$ in of because it is pronounced as a $/ \mathrm{v} /$ rather than as an $/ \mathrm{f} /$. There is no reason by that account for subjects told to search for the phoneme / $\mathrm{v} /$ to make a disproportionately large number of detection errors on the word of, because in this case the phoneme for which subjects are searching $(/ \mathrm{v} /)$ does occur in the test word.

Although Read's (1983) phonological recoding explanation cannot account for the present pattern of results, the mismatch between phoneme and letter representations may not be the only relevant factor. Visual word unitization processes are presumably contributing to this effect to some extent, because we also found a great tendency for subjects to make detection errors on the word of rela- tive to other words when searching for the vowel phoneme $/ 2 /$. However, word unitization processes cannot provide a complete account of this result, because the effect of test word was significantly smaller for the vowel than for the consonant target.

We found that when searching for the vowel phoneme $1 / 2 /$, the subjects missed $50 \%$ of the ofs but only $4 \%$ of the other test words. The magnitude of this effect is particularly surprising, given Read's (1983) finding that subjects made virtually no errors on the word of when they searched for the letter $o$ (in contrast to the very large percentage of errors when they searched for the letter $f$ ). In the earlier study by Schneider et al. (1991), unlike Read's, there was a significant difference between of and other words for $o$, but the difference in that case $(16 \%$ errors on of and $2 \%$ errors on other words in Experiment 3B) was much smaller than the present difference when subjects searched for the phoneme / $/$ /. In Experiment 3 , we addressed possible explanations for the relatively low error rate for the target $o$.

\section{EXPERIMENT 2}

Although the results of Experiment 1 provided support for the hypothesis that a mismatch between phoneme and letter representations of a target in a word leads to detection errors, visual word unitization processes were also implicated in the results. To eliminate the influence of visual unitization processes and thereby to highlight the effects of a mismatch between the phoneme and letter representations of a target, we used the auditory (rather than the visual) modality for the presentation of the passages in Experiment 2. (As before, however, all instructions, including those concerning the target phoneme, were presented visually.) Schneider et al. (1991) found that with the auditory modality subjects made more errors on of than on other words when they searched for the letter $f$, for which there was a mismatch between the phoneme and letter representations, but not when they searched for the letter $o$, for which there was no mismatch. Moreover, in studies of phoneme monitoring of auditorily presented text, responses are, if anything, facilitated (i.e., responses are faster) when the targets are embedded in familiar words rather than nonwords (see, e.g., Cutler, Mehler, Norris, \& Segui, 1987; Frauenfelder, Segui, \& Dijkstra, 1990; Rubin, Turvey, \& Van Gelder, 1976). Hence, it was predicted that subjects in the present experiment would make a significantly greater proportion of errors on of than on other words when searching for the consonant phoneme (because of the mismatch between the phoneme and letter representations) but not when searching for the vowel phoneme (because there is no mismatch in that case).

\section{Method}

Subjects. Forty-four undergraduate students taking a course on introductory psychology participated as subjects. There were equal numbers of subjects in the two counterbalancing groups. All were native speakers of English who had not participated in similar ex- 
periments previously. Sixteen additional subjects were tested, but their data were not analyzed: 11 of them made two or more false positive responses, and the remaining 5 were randomly selected to be dropped in order to equate the numbers of subjects in each group.

Apparatus and Materials. An Optisonics Corporation cassette tape recorder/player was used for the auditory presentation of the passages. The same passages were employed as in Experiment 1. They were spoken by a female at a slow speaking rate (the duration of each passage was $22 \mathrm{sec}$; thus, the rate was 1.18 words per second) using normal intonation.

Procedure. The subjects were tested in several small groups. As in Experiment 1, each subject participated in both a vowel and a consonant condition; the order of conditions was counterbalanced across subjects. The conditions were the same as in Experiment 1, except instead of being shown the phonetic symbol for the schwa vowel $/ 2 /$, the subjects were shown the phonetic spelling $/ \mathrm{uh} /$ in an attempt to avoid the apparent confusion by some subjects in Experiment 1 (those whose data were eliminated because of an excessive number of false positive responses) concerning the definition of the schwa phoneme. The passages were presented in the same order as in Experiment 1.

An instruction sheet preceded each passage. The subjects were instructed to listen to the passage on the tape recorder, and any time they heard a word that contained the target sound, they were to write the word in the space below the dotted line. As in Experiment 1 , they were also told that if they realized that they had missed a word with the target sound in it, they should not go back to that word. Further, they were reminded that the sound could occur anywhere in the word.

Design. The design was the same as in Experiment 1.

\section{Results}

The results are summarized in Table 2 in terms of the proportion of times out of the number possible that the subjects missed a target phoneme as a function of target and test word. Table 2 does not include a breakdown by the between-subjects counterbalancing factor of target order, because it did not enter into a significant interaction with test word. There was, however, a significant interaction of target order and target $\left[F(1,42)=10.2, M S_{\mathrm{e}}=\right.$ $0.0616, p<.01$ ], reflecting the fact that subjects made fewer errors on the second target (vowel first, vowel = .205 , consonant $=.074$; consonant first, vowel $=.142$, consonant $=.250$ ).

As in previous experiments, there was a large increase in detection errors for of relative to other words. Most importantly, the difference between of and other words was much larger for the consonant than for the vowel. A mixed analysis of variance yielded a significant main effect of test word $\left[F(1,42)=15.7, M S_{\mathrm{e}}=0.0761, p<\right.$ $.001]$, and a significant interaction of test word and target $\left[F(1,42)=5.0, M S_{\mathrm{e}}=0.0728, p<.05\right]$.

Separate planned analyses were conducted on the data for each target. The effect of test word was significant

Table 2

Proportion of Targets Missed in Experiment 2 as a Function of Target and Test Word

\begin{tabular}{ccc} 
& \multicolumn{2}{c}{ Test Word } \\
\cline { 2 - 3 } Target & of & Other Words \\
\hline$/ \mathrm{v} /$ & .290 & .034 \\
$/ \mathrm{a} /$ & .210 & .136 \\
\hline
\end{tabular}

for the consonant target $\left[F(1,42)=18.8, M S_{\mathrm{e}}=0.0766\right.$, $p<.001]$, but not for the vowel target $[F(1,42)=1.7$, $\left.M S_{\mathrm{e}}=0.0723, p>.10\right]$. For the consonant target there was also a significant main effect of target order $[F(1,42)$ $\left.=10.3, M S_{\mathrm{e}}=0.0665, p<.01\right]$, as well as a significant interaction of test word and target order $[F(1,42)=$ $\left.4.9, M S_{\mathrm{e}}=0.0766, p<.05\right]$, reflecting the fact that the effect of test word was greater when the consonant came first (vowel first, of $=.136$, other $=.011$; consonant first, of $=.443$, other $=.057$ ).

\section{Discussion}

The overall error rate in Experiment 2 was considerably lower than that in Experiment 1 . This finding is presumably due in part to the elimination of visual unitization processes in Experiment 2. However, previous research has shown that the overall error rate with auditorily presented text also depends strongly on the rate of presentation (Schneider et al., 1989). Hence, the effects of eliminating visual unitization processes are exhibited most clearly in the present experiment by comparing the error proportions on of and other words. When searching for the vowel target, subjects did not make significantly more errors on of than on other words, as had been expected because visual unitization processes were eliminated by the use of the auditory modality (see Schneider et al., 1989; Schneider et al., 1991). In contrast, when searching for the consonant target, subjects made many more detection errors on of than on other words, in agreement with the hypothesis that detection errors occur when there is a mismatch between the phoneme and letter representations of the target in a word.

\section{EXPERIMENT 3}

In Experiment 1, subjects made a considerable proportion of errors on of even when searching for the vowel target. Although the effect of test word was significantly larger for the consonant than for the vowel target in Experiment 1 , it was still substantial for the vowel target. In contrast, the effect of test word, although significant in some cases, was relatively small in earlier studies when subjects searched for the letter $o$ as the target (see, e.g., Schneider et al., 1991). Perhaps some specific properties of the letter $o$, such as its goodness of pattern (see, e.g., Garner \& Clement, 1963), or the well-formed Gestalt properties of its visual configuration, make it very easy to detect, even when embedded in a familiar word. Alternatively, the location of the letter $o$ at the start of the test word of may be an important factor contributing to the low error rate. It is also possible that the effect of test word was relatively small for the letter $o$ because it is a vowel rather than a consonant. However, earlier studies, in which very large effects of test word with the vowel target $e$ were found (see, e.g., Corcoran, 1966), make this explanation less plausible.

The aim of Experiment 3 was to examine the relative contributions to letter detection of three factors: (1) the familiarity of the test word, (2) the location of the target 
letter in the test word, and (3) the specific properties of the target letter. Toward that end, we compared the target letters $t$ and $o$ in three critical test words: $t o, t w o$, and not. The comparison of the target letters in the test words to and two allows for an assessment of the effects of test word familiarity when target location in the word and pronunciation are controlled. If word familiarity is a crucial factor, subjects should miss more targets in the common word to than in the less common word two. If the location of the target at the start of a two-letter word was responsible for the relatively small number of errors on the word of with the target $o$, we would expect fewer detection errors on $t$ than on $o$ in both test words to and two. Alternatively, if the specific properties of the letter $o$ were responsible for the relatively low error rate on the word of (e.g., if the well-formed Gestalt properties of its configuration make the $o$ easy to detect), we would expect fewer detection errors on $o$ than on $t$ in both the test words to and $t w o$. Further, because $t$ precedes $o$ in the test word to but $t$ follows $o$ in the test word not, the comparison of the target letters in the test words to and not allows for a different type of assessment of the relative effects of the location and the specific properties of the target letter. If the location of the target is of crucial importance, there should be a significant interaction of target letter and test word when the words to and not are compared. Alternatively, if the specific properties of the target are crucial, there should be a main effect of target letter, with more detection errors on $t$ than on $o$ in both test words to and not. (Note that the two sets of comparisons are not orthogonal; they overlap because both involve the test word to: to vs. two and to vs. not.) In this experiment, we employed the more standard version of the letter detection task (e.g., Healy, 1976), in which subjects read text and encircle target letters. The standard version of the task was not employed in the previous experiment because it cannot be used when the text is presented auditorily.

\section{Method}

Subjects. Thirty-six undergraduate students taking a course on cognitive psychology participated as subjects on the 1st day of their course before the presentation of any material on cognitive psychology. There were equal numbers of subjects in the two counterbalancing groups.

Materials. A 71-word passage was constructed; it included 32 instances of the target $o$ and 20 instances of the target $t$, typed with Courier 10-pitch font (produced by a laser printer connected to a VAX computer). There were two instances of each of the critical test words $t o, t w o$, and not. Note that each of these words contains both of the target letters. The words $t o$ and $t w o$ are identical in their pronunciation (although the vowel in to is often reduced to a schwa) but different in their frequency, with to much more common than two; $t o$ has frequency 26,149 and two has frequency 1,412 according to the norms of KuCera \& Francis (1967), which are based on a corpus of 1,014,232 words. For both of these words, the letter $t$ occurs in the first position and the letter $o$ in the last position. The test words $t o$ and not are more similar in frequency (not has frequency 4,609 according to Kučra \& Francis, 1967) but are dissimilar in pronunciation. For $t o$, the letter $t$ occurs before the letter $o$, but for not, the letter $o$ occurs before the letter $t$. The passage was, "I am pleased to see you in my course on Cognitive Psych.
Although one or two of you may not believe me from now on, the things you learn in the class will be valuable for the rest of your life. This is not to say all you learn from this course will be helpful for all time, but more than one or two things should improve the way you live."

Procedure. The subjects were tested in a single group session. Each subject participated in two conditions; the order of conditions was counterbalanced across subjects. The conditions differed only in the target, which was the letter $t$ or the letter $o$. The same passage was used for both conditions; each subject saw the passage twice, but the subject was not told that the passage was repeated.

An instruction sheet preceded each passage. The subjects were instructed to read the passage at their normal reading speed, but whenever they came to an instance of the target letter (typed in either lowercase or uppercase), they were to encircle it. They were further told that if at any time they realized that they had missed a target in a previous word, they should not retrace their steps to encircle it. In addition, they were instructed that they were not expected to get every target, so they should not slow down their reading speed in order to be overcautious about getting the targets.

Design. The experimental design comprised two overlapping parts. For the first part, the design was a $2 \times 2 \times 2$ mixed factorial. For this part, only the test words two and to were scored for both target conditions. The first factor, target order, was a counterbalancing factor varied between subjects, and the other two factors were varied within subjects. The second factor was target $(o, t)$, and the third factor was test word $(t w o, t o)$. For the second part, the design was the same as for the first part, except that the test words were changed to include to and not.

\section{Results}

The results are summarized in Table 3 in terms of the proportion of times out of the number possible that the subjects missed a target letter as a function of target and test word. Table 3 does not include a breakdown by the between-subjects counterbalancing factor of target order, because it did not enter into any significant main effect or interactions.

The comparison of the test words two and to revealed both more errors on the more common than on the less common word and more errors for the target $t$ than for the target $o$. A mixed factors analysis of variance yielded significant main effects of test word $[F(1,34)=6.0$, $\left.M S_{\mathrm{e}}=0.0234, p<.05\right]$ and target $[F(1,34)=6.3$, $\left.M S_{\mathrm{e}}=0.0222, p<.05\right]$. The interaction of these two factors was not significant $\left[F(1,34)=2.3, M S_{\mathrm{e}}=\right.$ $0.0189, p>.10]$.

The comparison of the test words to and not revealed no difference between the two words, which differed in terms of the locations of each target letter. As in the previous comparison, however, more errors were made for the target $t$ than for the target $o$. Only the main effect of target was significant $\left[F(1,34)=14.6, M S_{\mathrm{e}}=0.0476\right.$,

Table 3

Proportion of Targets Missed in Experiment 3

for the Targets $t$ and $o$ as a Function of Test Word Test Word

\begin{tabular}{cccc} 
Target & two & to & not \\
\hline$o$ & .000 & .028 & .000 \\
$t$ & .028 & .125 & .181 \\
\hline
\end{tabular}


$p<.001]$ in the mixed factors analysis of variance. The interaction of test word and target was not significant $\left[F(1,34)=2.0, M S_{\mathrm{e}}=0.0313, p>.10\right]$.

\section{Discussion}

Our finding that subjects made more errors with the target $t$ than with the target $o$, both when $t$ preceded $o$ in the test word to and when $t$ followed $o$ in the test word not, implies that letter detection errors depend more on the specific properties of the target than on its location in the test word. For example, the well-formed Gestalt properties of the visual configuration of the letter $o$ may make it easy to detect. Further, our finding more errors on the more common word to than on the less common word two, in which comparison test word pronunciation and letter location in the word are controlled, suggests that word frequency also affects letter detection errors, presumably because word unitization is more likely with more common words. It is important to note, however, that the words to and two, although identical in pronunciation, differ in ways other than word frequency, and these other differences may also contribute to the effect of test word. Specifically, the words differ in length and in syntactic function. But both length and syntactic function have been controlled in other studies, in which significant effects of word frequency were nevertheless obtained. For example, Healy $(1976,1980)$ compared letter detection in common and rare nouns, equating the length of the words and the location of the target letters, and found significantly more errors on the common nouns. More recent investigators have found, however, that syntactic function does play an important role in letter detection (see Greenberg \& Koriat, 1991; Koriat \& Greenberg, 1991; Koriat, Greenberg, \& Goldshmid, 1991).

\section{EXPERIMENT 4}

In Experiment 3, we found that subjects made more errors with the target $t$ than with the target $o$, and we hypothesized that this difference could be explained in terms of the visual characteristics of the targets, such as the well-formed Gestalt properties of the target $o$. To test this hypothesis, in the present experiment we directly manipulated the visual characteristics of the targets by comparing two different type fonts, a standard font and an unusual font. Sanocki $(1987,1988)$ used similar manipulations in tasks involving the presentation of four-letter strings, and he found an advantage for simpler ("minimal") fonts relative to those with serifs. In the standard font that we used, the target $o$, but not the target $t$, is perfectly symmetrical and regular, whereas in the unusual font, the target $o$ is less symmetrical and less regular and the target $t$ is more symmetrical and more regular. That is, the unusual font distorted the configural properties of the letter $o$ but accentuated those for the letter $t$. If the visual characteristics of the target do affect the rate of detection errors, subjects should make more errors on the target $o$ and fewer errors on the target $t$ in the unusual font than in the regular font.

\section{Method}

Subjects. Twenty-four undergraduate students taking a course on introductory psychology participated as subjects. There were equal numbers of subjects in the two font groups and the two counterbalancing subgroups of each font group.

Materials. The same passage was employed as in Experiment 3. Two different versions of the passage were produced by a laser printer connected to a Macintosh computer. Each subject was shown only one version, with the version shown depending on the font group assigned to the subject. One version was typed with a standard font (Times 12 with New York 12 spacing). This font includes symmetrical $o$ and regular $t \mathrm{~s}$. The other version was typed with an unusual font (Nordic 12), which includes very asymmetrical os and $t$ that are more regular than in normal printing (see Figure 1). The words were located in the same relative positions on the page in the two versions of the passage.

The instructions for both conditions were typed with a third font (Chicago 12).

Procedure. The subjects were tested individually. Each subject participated in two conditions; the order of conditions was counterbalanced across subjects in each font group. The conditions differed only in the target, which was the letter $t$ or the letter $o$. The same passage was used for both conditions; each subject saw the passage twice, always in the same font, but the subject was not told that the passage was repeated.

An instruction sheet preceded each passage. The instructions were the same as those used in Experiment 3.

Design. The experimental design comprised two overlapping parts. For the first part, the design was a $2 \times 2 \times 2 \times 2$ mixed factorial. For this part, only the test words two and to were scored for both target conditions. The first two factors, font condition (standard, Nordic) and the counterbalancing factor of target order, were varied between subjects, and the other two factors were varied within

Times

I am pleased to see you in my course on Cognitive Psych.

Mordic

I am pleased to see you in my course on Cognitive psych.

Chicago

I am pleased to see you in my course on Cognitive Psych.

Figure 1. Fonts used in Experiment 4. First words from the passage used in Experiments 3 and 4 . Note that the Chicago font was used only for the instructions. 
subjects. The third factor was target $(o, t)$, and the fourth factor was test word $(f w o, t o)$. For the second part, the design was the same as for the first part, except that the test words were changed to compare to and not.

\section{Results}

The results are summarized in Table 4 in terms of the proportion of times out of the number possible that the subjects missed a target letter as a function of font condition, target, and test word. Table 4 does not include a breakdown by the between-subjects counterbalancing factor of target order, because it did not enter into any significant main effect or interactions.

As in Experiment 3, the comparison of the test words two and to revealed both more errors on the more common than on the less common word and more errors for the target $t$ than for the target $o$. The difference between targets was reduced with the Nordic font relative to the standard font. Furthermore, detection errors on the target $t$ were fewer for the Nordic font, which has a more regular $t$, than for the standard font, whereas detection errors on the target $o$ were greater for the Nordic font, which has a less regular $o$, than for the standard font. In addition, the effect of test word depended on target letter. The difference between the common and less common test words was greater for the target $t$ than for the target $o$. Most crucially, the effect of test word depended on the combination of target letter and font condition. The difference between common and less common test words in the Nordic font was just as large for the target $o$ as for the target $t$; it was only in the standard font that the effect of test word was greater for the target $t$ than for the target $o$. A mixed factors analysis of variance yielded significant main effects of test word $\left[F(1,20)=18.2, M S_{\mathrm{e}}=\right.$ $0.0573, p<.001]$ and target $\left[F(1,20)=14.8, M S_{\mathrm{e}}=\right.$ $0.0344, p<.01]$, and significant interactions of target and font condition $\left[F(1,20)=7.6, M S_{\mathrm{e}}=0.0344, p<\right.$ $.05]$, test word and target $\left[F(1,20)=5.5, M S_{\mathrm{e}}=0.0302\right.$, $p<.05]$, and test word, target, and font condition $\left[F(1,20)=12.4, M S_{\mathrm{e}}=0.0302, p<.01\right]$.

The comparison of the test words to and not revealed only a marginal difference between the two words, with a tendency for more errors on the more common word to. In agreement with Experiment 3, more errors were made for the target $t$ than for the target $o$. Importantly, there was an interaction between target and font condi-

\section{Table 4}

Proportion of Targets Missed in Experiment 4 for the Targets $t$ and $o$ as a Function of Font Condition and Test Word

\begin{tabular}{cccc}
\hline & \multicolumn{3}{c}{ Test Word } \\
\cline { 2 - 4 } Condition & two & to & not \\
\hline Standard font & & & \\
$o$ target & .000 & .042 & .000 \\
$t$ target & .042 & .500 & .375 \\
Nordic font & & & \\
$o$ target & .000 & .208 & .042 \\
$t$ target & .083 & .208 & .167 \\
\hline
\end{tabular}

tion. As in the comparison of the test words to and two, the difference between targets was reduced with the Nordic font relative to the standard font. Furthermore, detection errors on the target $t$ were fewer for the Nordic font, which has a more regular $t$, than for the standard font, whereas detection errors on the target $o$ were greater for the Nordic font, which has a less regular $o$, than for the standard font. A mixed factors analysis of variance yielded a marginally significant main effect of test word $\left[F(1,20)=3.2, M S_{\mathrm{e}}=0.0661, p<.10\right]$, a significant main effect of target $\left[F(1,20)=16.6, M S_{\mathrm{e}}=0.0828\right.$, $p<.001]$, and a significant interaction of font condition and target $\left[F(1,20)=9.1, M S_{\mathrm{e}}=0.0828, p<.01\right]$.

\section{Discussion}

As in Experiment 3, we found that subjects made more errors with the target $t$ than with the target $o$, which is consistent with the explanation that letter detection errors depend more on the specific properties of the target (e.g., the well-formed Gestalt properties of the visual configuration of the letter $o$ may make it easy to detect) than on its location in the test word. When the Gestalt properties of the letter $o$ were disturbed by the use of the Nordic font, detection errors on the letter $o$ increased, and when the Gestalt properties of the letter $t$ were enhanced by the use of the Nordic font, detection errors on the letter $t$ were decreased, further supporting this explanation. Indeed detection errors with the Nordic font on the common word to were just as high on the letter $o$ as on the letter $t$. Note that this finding also underlines the fact that the differences between the results with the targets $o$ and $t$ cannot be explained simply by referring to the fact that the letter $o$ is a vowel and the letter $t$ is a consonant.

\section{EXPERIMENT 5}

The results of Experiment 4 indicate that letter detection performance is enhanced when the Gestalt properties of a letter are enhanced and disturbed when the Gestalt properties are disturbed. These findings are clear and can easily be interpreted in terms of the visual configurations of the letters, but the findings are less clear and harder to interpret in terms of the visual configurations of the larger word units. Specifically, the unusual Nordic font not only disturbs the visual configuration of the letter $o$ but also disturbs the visual configuration of the words containing the targets. Because earlier studies with alternating type case (e.g., tHe, Drewnowski \& Healy, 1977) or interpolated asterisks (e.g., ${ }^{*}{ }^{*}{ }^{*}{ }^{*} d^{*}$, Healy, Conboy, \& Drewnowski, 1987) indicated that disturbing the familiar configuration of very common words decreased letter detection errors, it might be expected that using an unusual font would also lead to a decrease in letter detection errors on very common words. Examination of the error rate on the common word to yields conflicting evidence for that prediction; although the error rate did decrease in the Nordic font relative to the standard font for the target $t$, it increased in the Nordic font relative to the 
standard font for the target $o$, in accordance with the predictions based on the changes in the visual configurations of the target letters.

The aim of Experiment 5 was to provide a more sensitive test of the hypothesis that an unusual font that disturbs the visual configuration of word units should lead to a decrease in letter detection errors in very common test words. To increase the sensitivity of the test of the word unitization hypothesis, we returned in this experiment to the very common test word of, especially because we had evidence from Experiment 1 as well as from other previous studies (e.g., Schneider et al., 1991) that visual word unitization processes apply to the common word of. Also, to increase the sensitivity of the test of the word unitization hypothesis, we changed to a script font that disturbed the visual configuration of the letters more uniformly than did the Nordic font (which disturbed the visual configuration of some letters but not others). The script font also had the advantage of more clearly disturbing the visual configuration of the entire word units.

We used both the letters $o$ and $f$ as targets, as in the study by Schneider et al. (1991). According to the word unitization hypothesis, letter detection errors should decrease on the word of (and less so on other words) in the script font relative to the standard font for both target letters. On the other hand, the target letter $o$, but not the target letter $f$, has well-formed Gestalt properties in the standard font that are disturbed by the script font. The results of Experiment 4 suggest that this disturbance should lead to an increase in letter detection errors for the $o$ target, which should counteract the decrease in letter detection errors predicted by the word unitization hypothesis to some extent. More specifically, according to the unitization account of performance in the letter detection task (see, e.g., Healy, Oliver, \& McNamara, 1987), as reviewed earlier, processing of text occurs in parallel at the level of the word and at the level of the letter. Familiarity of a word unit facilitates its processing. Once a word unit has been identified, subjects proceed to the next segment of text, even if they have not yet identified all of the component letters in the word. Letter detection errors occur when the word containing a target letter is identified before the target letter itself is identified. This account can accommodate the finding that more letter detection errors generally occur with the target $f$ than with the target $o$ if the visual properties of the letter $o$, such as its well-formed Gestalt goodness of pattern, allow for it to be identified more rapidly than the letter $f$, so that the letter $o$ is more likely to be identified than the letter $f$ before the word that contains it is identified. By this account, letter detection errors should increase when the visual properties of the letter are disturbed by making its configuration less regular and thus slowing down its identification. Conversely, letter detection errors should decrease when the visual properties of the word are disturbed by making its configuration less familiar and thus slowing down its identification, thereby allowing more time for the identification of its component letters. Hence, the script font should disturb, or slow down, the identification of both the common word of and its component letter $o$. For the target $f$, these disturbances should lead to an overall decrease in letter detection errors. In contrast, for the target $o$, these two disturbances should have opposite effects in terms of detection errors; the disturbance of the word configuration should lead to a decrease in detection errors, and the disturbance of the letter configuration should lead to an increase in detection errors. In every case, however, whether an error occurs for a given subject on a given letter depends on whether the letter is identified before the specific word.

In addition to these visual factors that influence letter detection, there are phonetic factors that should affect the error rate in the letter detection task. Specifically, the target letter $f$, but not the target letter $o$, is pronounced in an unusual manner in the test word of, which should lead to relatively more detection errors on the word of for the letter $f$ than for the letter $o$ in both type fonts. Hence, combining predictions from the word unitization, letter Gestalt, and letter-phoneme cross-checking hypotheses leads to the expectation of a three-way interaction between font condition, test word, and target letter. In particular, a large decrease in letter detection errors is expected on the common word of, and less so on other words, for the script font relative to the standard font with the target $f$. A smaller decrease, or perhaps even an increase, is expected with the target $o$.

\section{Method}

Subjects. Twenty-four undergraduate students who were taking a course on introductory psychology participated as subjects.

Materials. A 68-word passage used in a previous study (Schneider et al., 1991, Experiment 2) was employed here. The passage included 18 instances of the targets $f$ and $o$. There were 9 instances of the test word of and 9 other test words, each of which contained a single instance of both target letters. The passage was, "Last Wednesday a soft ball of gum came from the back of the small loft and hit the teacher in the side of his head. Yesterday four of the students went before the rest of the class in front of the teacher and said that they knew things of this type happened often, but all of them must forget what they saw for the rest of their lives."

The passage was typed in two different fonts produced on a Macintosh computer. The first was a standard font that consisted of New York 12 characters (TrueType), and the second was a script font that consisted of Florence 12 characters (see Figure 2).

Procedure. The subjects were tested individually. Each subject participated in one font condition only, but with both target letters in counterbalanced order. The font conditions differed only in the font in which the passage was typed.

Each subject saw the same passage twice, once with instructions to detect the letter $o$ and once with instructions to detect the letter $f$. Each presentation of the passage was preceded by an instruction sheet. The instructions were typed in a third font (Chicago 12) not identical to either the standard or the script font. The subjects were given the same instructions as in Experiments 3 and 4 (except for the targets).

Design. The design was a $2 \times 2 \times 2 \times 2$ mixed factorial. The first two factors, font condition (standard or script font) and the counterbalancing factor of target letter order ( $o$ first or $f$ first), were varied between subjects. The last two factors, test word (of and other words) and target letter ( $o$ and $f$ ), were varied within subjects. 
New York

Last Wednesday a soft ball of gum came from the

7larence

Last Mednesday a soft ball of gum came from the

Chicago

Last Wednesday a soft ball of gum came from the

Figure 2. Fonts used in Experiments 5 and 6. First words from the passage used in Experiment 5 . Note that the Chicago font was used only for the instructions.

\section{Results}

The results are summarized in Table 5 in terms of the proportion of times out of the number possible that the subjects missed a target letter as a function of font condition, target letter, and test word. Table 5 does not include a breakdown by the counterbalancing factor of target letter order because that factor did not yield a significant main effect and did not enter into any significant interactions. As in previous experiments and in accordance with the word unitization and letter-phoneme crosschecking hypotheses, subjects made more errors on the common word of than on other less familiar words and more errors with the $\operatorname{target} f$ than with the target $o$. Also in accordance with the word unitization hypothesis, subjects made more errors in the standard condition than in the script condition. Further, as found previously and in accordance with the letter-phoneme cross-checking hypothesis, the difference between errors on of and errors on other words was greater for the target $f$ than for the target $o$. Importantly, in accordance with the word unitization hypothesis, the effect of test word was diminished in the script condition. In addition, in accordance with the letter Gestalt hypothesis, the difference between the targets $o$ and $f$ was diminished in the script condition, and test word, target letter, and font condition interacted so that the effect of test word was greatest by far for the target $f$ in the standard condition. A mixed factors analysis of variance yielded significant main effects of test word $\left[F(1,20)=34.3, M S_{\mathrm{e}}=0.0454, p<.001\right]$, target letter $\left[F(1,20)=62.3, M S_{\mathrm{e}}=0.0101, p<.001\right]$, and font condition $\left[F(1,20)=11.6, M S_{\mathrm{e}}=0.0425, p<.01\right]$, as well as significant interactions of test word and target letter

Table 5

Proportion of Targets Missed in Experiment 5 as a Function of Font Condition, Target Letter, and Test Word

\begin{tabular}{ccc}
\hline as a Function of Font Condition, Target Letter, and Test Word \\
\cline { 2 - 3 } Condition & of & Test Word \\
\hline Standard font & & Other Words \\
$o$ target & .176 & .092 \\
$f$ target & .731 & .065 \\
Script font & & \\
$o$ target & .120 & .065 \\
$f$ target & .259 & .046 \\
\hline
\end{tabular}

$\left[F(1,20)=49.9, M S_{\mathrm{e}}=0.0165, p<.001\right]$, test word and font condition $\left[F(1,20)=7.7, M S_{\mathrm{e}}=0.0454, p<\right.$ $.05]$, target letter and font condition $[F(1,20)=24.6$, $\left.M S_{\mathrm{e}}=0.0101, p<.001\right]$, and test word, target letter, and font condition $\left[F(1,20)=16.5, M S_{\mathrm{e}}=0.0165\right.$, $p<.001]$.

\section{Discussion}

Combining predictions from the word unitization, letter Gestalt, and letter-phoneme cross-checking hypotheses led us to predict a specific three-way interaction between font condition, test word, and target letter, and that specific interaction was, indeed, obtained. In particular, the letter detection errors decreased for the script font relative to the standard font, especially on the common word of with the target letter $f$. These findings reemphasize the importance of word unitization processes in letter detection and demonstrate that font changes influence processing at the word level as well as at the level of the individual letter.

\section{EXPERIMENT 6}

In Experiment 5, as in previous studies (e.g., Schneider et al., 1991), we found that subjects made a large proportion of detection errors on the common word of when searching for the target letter $f$. This proportion was decreased greatly when a script font was used instead of a standard font. The decrease was attributed largely to the disruption of word unitization processes. However, as was clear from the present Experiments 1 and 2 and the earlier studies by Read (1983) and Schneider et al. (1991), letter detection errors on the word of are also influenced by factors other than word unitization (including letter-phoneme cross-checking). To provide a clearer test of the word unitization hypothesis (according to which an unusual font that disturbs the visual configuration of entire word units should lead to a decrease in letter detection errors in common test words), we turned to the very common word the, because letter detection errors on that word have been found to be very sensitive to word unitization and relatively insensitive to other factors (see, e.g., Drewnowski \& Healy, 1977; Healy, 1976; Healy, Conboy, \& Drewnowski, 1987; Schneider et al., 1989). Specifically, 
we used the target $t$ with a prose passage including instances of the common word the as well as less common test words; the passage was typed with the two fonts used in Experiment 5. As in previous studies (e.g., Healy, 1976), we expected a large proportion of errors on the relative to that on the less common words. According to the word unitization hypothesis, the proportion of errors on the, and to a lesser extent on other words, should decrease with the script font in comparison with the standard font because the script font should disturb the familiar visual configuration of the test words.

\section{Method \\ Subjects. Fifty undergraduate students who were taking a course on introductory psychology participated as subjects. \\ Materials. A 25-word passage used in a previous study (Schneider et al., 1989) was employed here. The passage included 12 instances of the target $t$. There were 4 instances of the test word the and 8 other test words, each of which contained a single instance of the target letter. The passage was, "The boys' mother cleaned their clothes the other day. There was soap on the floor. Then Bob's brother slipped on the slick lather and fell." \\ As in Experiment 5, the passage was typed in two different fonts produced on a Macintosh computer. The first was a standard font that consisted of New York 12 characters (TrueType), and the sec- ond was a script font that consisted of Florence 12 characters. \\ Procedure. The subjects were tested individually. Each subject participated in one font condition only. The font conditions differed only in the font in which the passage was typed. \\ An instruction sheet preceded the passage. As in Experiment 5, the instructions were typed in a third font (Chicago 12) not identi- cal to either the standard or the script font. The subjects were given the same instructions as in Experiments 3, 4, and 5 (except for the target).}

Design. The design was a $2 \times 2$ mixed factorial. The first factor, font condition (standard or script font), was varied between subjects. The second factor, test word (the or other), was varied within subjects.

\section{Results}

The results are summarized in Table 6 in terms of the proportion of times out of the number possible that the subjects missed a target letter as a function of font condition and test word. As in previous studies, subjects made more errors on the common word the than on other less familiar words. As in Experiment 4, they also made more errors in the standard condition than in the script condition. Importantly, the effect of test word was diminished in the script condition. A mixed factors analysis of variance yielded significant main effects of font condition $\left[F(1,48)=9.3, M S_{\mathrm{e}}=0.0341, p<.01\right]$ and test word $\left[F(1,48)=49.0, M S_{\mathrm{e}}=0.0276, p<.001\right]$, as well as a significant interaction of font condition and test word $\left[F(1,48)=8.6, M S_{\mathrm{e}}=0.0276, p<.01\right]$.

Table 6

Proportion of Targets Missed in Experiment 6 as a Function of Font Condition and Test Word

\begin{tabular}{lcc}
\hline & \multicolumn{2}{c}{ Test Word } \\
\cline { 2 - 3 } Condition & the & Other Words \\
\hline Standard font & .370 & .040 \\
Script font & .160 & .025 \\
\hline
\end{tabular}

\section{Discussion}

As expected, the proportion of letter detection errors on the word the was decreased substantially with a script font relative to a standard font. This decrease is consistent with the word unitization hypothesis and with other studies (e.g., Drewnowski \& Healy, 1977; Healy, 1976; Healy, Conboy, \& Drewnowski, 1987; Schneider et al., 1989) showing evidence for the effects of word unitization on letter detection errors in the word the.

\section{GENERAL DISCUSSION}

On the basis of previous detection experiments, Schneider et al. (1991) suggested that the mental lexicon includes both phoneme and letter representations and that there is a cross-checking or communication between these two types of representations. The primary evidence for this suggestion was the finding that subjects make more detection errors on the word of than on other words when searching for the letter $f$. This suggestion leads to the prediction, which was verified in the present Experiments 1 and 2 , that subjects should also make more detection errors on the word of than on other words when searching for the phoneme $/ v /$. This finding, which was obtained with a single target, is not consistent with the possibility that the mismatching effect only occurs when subjects are given a combined target consisting of two phonemes (Schneider et al., 1991) and are, thus, led to make a comparison of the two phonemes. This finding is also inconsistent with the phonological recoding explanation proposed by Read (1983), and it cannot be attributed solely to visual unitization processes or to the high frequency of the word of, because it occurred even when the passage was presented auditorily and because the difference in detection errors on of and on other words was not significant when subjects searched for the vowel phoneme $/ 2 /$ with auditory presentation in Experiment 2 . However, visual word unitization processes and word frequency were also shown to have important influences on the rate of detection errors by the findings that a large difference in detection error rate between of and other words occurred when subjects searched for the vowel phoneme /a/ with visual presentation in Experiment 1 and that more detection errors occurred in Experiments 3 and 4 for both the letter $t$ and the letter $o$ in the common word $t o$ than in the less common word two, even though the phonemes were equivalent in both words. In addition, in Experiments 5 and 6 , it was found that the large proportions of letter detection errors on the common words of and the relative to those on less common words were decreased substantially when the familiar visual configuration of the words was altered by using a script, rather than a standard, type font.

Another factor that must be considered when one is comparing detection errors on the letters $o$ and $f$ or on the phonemes $/ a /$ and $/ v /$ in the word of is their relative locations in the word. This factor was examined in Experiments 3 and 4 by comparing detection errors on the letters $t$ and $o$ in the words $t o$ and not, which differ in 
the relative locations of the two letters. Although $t$ precedes $o$ in the word to but follows $o$ in the word not, subjects made significantly more errors on $t$ than on $o$ in both words. This difference between the two letters may be attributed to specific properties of the letters, such as their visual configurations. The specific pronunciations of the letters may also be relevant, but it is interesting to note that subjects made essentially no errors on the letter $o$ in Experiment 3 despite the fact that it represented different phonemes in the words to and not.

The importance of the visual configurations of the letters was demonstrated by the manipulations of type font in Experiment 4 . When the Gestalt goodness of the visual pattern of the letter $o$ was disturbed by using the unusual Nordic font, detection errors increased. Likewise, when the Gestalt goodness of the visual pattern of the letter $t$ was enhanced by using the unusual Nordic font, detection errors decreased.

One of the most striking observations made in earlier studies of letter detection was the very small error rate on the word of when the letter $o$ was the target in contrast to the very large error rate on the word of when the letter $f$ was the target. In fact, Read (1983) found performance was close to perfect on the word of when subjects searched for the letter $o$. Schneider et al. (1991) found that subjects made significantly more errors on of than on other words when searching for the target $o$, but the difference was relatively small in that case $(16 \%$ errors on of and 2\% errors on other words in Experiment 3B). This observation of a very low error rate on of for the target $o$ was used previously by both Read (1983) and Schneider et al. (1991) to provide crucial support for theoretical proposals concerning the importance of phonetic processes in letter detection. Two contrasting observations in the present study underline the importance of visual processes in the same paradigm. First, in Experiment 1 , when subjects searched for the vowel phoneme $/ 2 /$ in visually presented text, the error rate on of was substantially greater than that on other words $(50 \%$ errors on of and 4\% errors on other words), whereas in Experiment 2 , when subjects searched for the vowel phoneme $/ a /$ in auditorily presented text, the error rate on of was not significantly greater than that on other words $(21 \%$ errors on of and $14 \%$ errors on other words). Second, in Experiment 4, the percentage of errors when subjects searched for the letter $o$ increased significantly when a nonstandard type font was employed. In fact, there was no difference in detection errors on the common word to between the targets $o$ and $t$ with the Nordic font. Further support for the importance to letter detection of visual processes, in general, and type font, in particular, comes from Experiments 5 and 6, which demonstrated a reduction in letter detection errors, especially on common words, when the visual configuration of the words was disturbed by using a script font instead of a standard type font. The effects of type font in Experiment 4 are attributable to visual processes operating at the letter level, whereas the effects of type font in Experiments 5 and 6 are attribut- able in large part to visual processes operating at the word level.

The combined observations from the experiments in this study, along with those from previous studies, indicate that both phonetic and visual processes influence letter detection and that the visual processes operate at both the letter and word levels. A full understanding of performance in the letter detection task must, therefore, include two types of processing-visual and phonetic-and the visual processing must include at least two levels-letters and words. It is not clear how many or which levels are involved in the phonetic processing. Because syllabic stress patterns were found to influence letter detection errors, Drewnowski and Healy (1982) argued that phonetic units at the level of the syllable influence letter detection. However, phonetic processes, like visual ones, may operate at more than one level in the letter detection task. Schneider et al. (1991) suggested that the visual and phonetic processes interact or communicate by means of a cross-checking process. The existence of such a process would imply that a phoneme level is involved, perhaps in addition to the syllable level. The results of the present Experiments 1 and 2 provide further evidence for a cross-checking process by demonstrating the large error rate on the word of relative to that on other words when subjects searched for the consonant phoneme $/ \mathrm{v} /$, even when the text was presented auditorily. It therefore seems advisable when one is developing a model of letter and word identification that one include a cross-checking process to provide for a type of communication between phonetic and visual information. Further research is needed to delineate the precise nature of this crosschecking process and the levels of phonetic and visual information on which the process operates.

\section{REFERENCES}

Corcoran, D. W. J. (1966). An acoustic factor in letter cancellation. Nature, 210, 658.

Cutler, A., Mehler, J., Norrs, D., \& Segui, J. (1987). Phoneme identification and the lexicon. Cognitive Psychology, 19, 141-177.

Drewnowski, A., Healy, A. F. (1977). Detection errors on the and and: Evidence for reading units larger than the word. Memory \& Cognition, 5, 636-647.

Drewnowski, A., Healy, A. F. (1982). Phonetic factors in letter detection: A reevaluation: Memory \& Cognition, 10, 145-154.

Frauenfelder, U. H., Segui, J., \& Dukstra, T. (1990). Lexical effects in phonemic processing: Facilitatory or inhibitory? Journal of Experimental Psychology: Human Perception \& Performance, 16, $77-91$.

GARNER, W. R. (1974). The processing of information and structure. Hillsdale, NJ: Erlbaum.

Garner, W. R., \& Clement, D. E. (1963). Goodness of pattern and pattern uncertainty. Journal of Verbal Learning \& Verbal Behavior, 2, 446-452.

Greenberg, S. N., \& Koriat, A. (1991). The missing-letter effect for common function words depends on their linguistic function in the phrase. Journal of Experimental Psychology: Learning, Memory, \& Cognition, 17, 1051-1061.

Healy, A. F. (1976). Detection errors on the word the: Evidence for reading units larger than letters. Journal of Experimental Psychology: Human Perception \& Performance, 2, 235-242.

Healy, A. F. (1980). Proofreading errors on the word the: New evi- 
dence on reading units. Journal of Experimental Psychology: Human Perception \& Performance, 2, 573-579.

Healy, A. F., Conboy, G. L., \& Drewnowski, A. (1987). Characterizing the processing units of reading: Effects of intra- and interword spaces in a letter detection task. In B. K. Britton \& S. M. Glynn (Eds.), Executive control processes in reading (pp. 279-296). Hillsdale, NJ: Erlbaum

Healy, A. F., Oliver, W. F., \& McNamara, T. P. (1987). Detection errors in continuous text: Effects of display size. Journal of Experimental Psychology: Human Perception \& Performance, 13, 279-290.

Koriat, A., \& Greenberg, S. N. (1991). Syntactic control of letter detection: Evidence from English and Hebrew nonwords. Joumal of Experimental Psychology: Learning, Memory, \& Cognition, 17, $1035-1050$.

Koriat, A., Greenberg, S. N., \& Goldshmid, Y. (1991). The missingletter effect in Hebrew: Word frequency or word function? Journal of Experimental Psychology: Learning, Memory, \& Cognition, 17, 66-80.

KRUEGER, L. E. (1992). The word-superiority effect and phonological recoding. Memory \& Cognition, 20, 685-694.

KuCERA, H., \& Francis, W. N. (1967). Computational analysis of present-day American English. Providence, RI: Brown University Press.

LABerge, D., \& SAmuels, S. J. (1974). Toward a theory of automatic information processing in reading. Cognitive Psychology, 6, 293-323.

MASSARO, D. W. (1987). Speech perception by ear and eye: A paradigm for psychological inquiry. Hillsdale, NJ: Erlbaum.

MCGURK, H., \& MACDONALD, J. (1976). Hearing lips and seeing voices: A new illusion. Nature, 264, 746-748.
READ, J. D. (1983). Detection of Fs in a single statement: The role of phonetic recoding. Memory \& Cognition, 11, 390-399.

Rubin, P., Turvey, M. T., \& Van Gelder, P. (1976). Initial phonemes are detected faster in spoken words than in spoken nonwords. Perception \& Psychophysics, 19, 394-398.

SANOCKI, T. (1987). Visual knowledge underlying letter perception Font-specific, schematic tuning. Joumal of Experimental Psychology: Human Perception \& Performance, 13, 267-278.

SANOCKI, T. (1988). Font regularity constraints on the process of letter recognition. Joumal of Experimental Psychology: Human Perception \& Performance, 14, 472-480.

Schneider, V. I., Healy, A. F., Ericsson, K. A., \& Bourne, L. E. (1989). Letter detection errors in reading, auditory, and memory tasks. Joumal of Memory \& Language, 28, 400-411.

Schneider, V. I., Healy, A. F., \& Gesi, A. T. (1991). The role of phonetic processes in letter detection: A reevaluation. Journal of Memory \& Language, 30, 294-318.

Seidenberg, M. S., \& Tanenhaus, M. K. (1979). Orthographic effects on rhyme monitoring. Journal of Experimental Psychology: Human Learning \& Memory, 12, 315-328.

VAN ORDEN, G. C. (1987). A ROWS is a ROSE: Spelling, sound, and reading. Memory \& Cognition, 15, 181-198.

VENEZKY, R. L. (1970). The structure of English orthography. The Hague: Mouton.

(Manuscript received July 8, 1992; revision accepted for publication April 9, 1993.) 